\title{
Regularization of closed positive currents of type $(1,1)$ by the flow of a Chern connection
}

\author{
Jean-Pierre Demailly \\ Université de Grenoble I, Institut Fourier, BP 74 \\ U.R.A. 188 du C.N.R.S., F-38402 Saint-Martin d'Hères
}

Dedicated to Professor Pierre Dolbeault on the occasion of his retirement

\section{Introduction}

Let $X$ be a compact $n$-dimensional complex manifold and let $T$ be a closed positive current of bidegree $(1,1)$ on $X$. In general, $T$ cannot be approximated by closed positive currents of class $C^{\infty}$ : a necessary condition for this is that the cohomology class $\{T\}$ is numerically effective in the sense that $\int_{Y}\{T\}^{p} \geq 0$ for every $p$-dimensional subvariety $Y \subset X$. For example, if $E \simeq \mathbb{P}^{n-1}$ is the exceptional divisor of a one-point blow-up $X \rightarrow X^{\prime}$, then $T=[E]$ cannot be positively approximated: for every curve $C \subset E$, we have $\int_{C}\{E\}=\int_{C} c_{1}(\mathcal{O}(-1))<0$. However, we will see that it is always possible to approximate a closed positive current $T$ of type $(1,1)$ by closed real currents admitting a small negative part, and that this negative part can be estimated in terms of the Lelong numbers of $T$ and the geometry of $X$.

Let $\alpha$ be a smooth closed $(1,1)$-form representing the same $\partial \bar{\partial}$-cohomology class as $T$ and let $\psi$ be a quasi-psh function on $X$ (that is, a function which is locally the sum of a plurisubharmonic function and a smooth function) such that $T=\alpha+\frac{i}{\pi} \partial \bar{\partial} \psi$. Such a decomposition exists even when $X$ is non-Kähler, since we can always find an open covering $\left(\Omega_{k}\right)$ of $X$ such that $T=\frac{i}{\pi} \partial \bar{\partial} \psi_{k}$ over $\Omega_{k}$, and construct a global $\psi=\sum \theta_{k} \psi_{k}$ by means of a partition of unity $\left(\theta_{k}\right)$ (note that $\psi-\psi_{k}$ is smooth on $\Omega_{k}$ ). If $\psi_{\varepsilon}$ is an approximation of $\psi$, then $T_{\varepsilon}=\alpha+\frac{i}{\pi} \partial \bar{\partial} \psi_{\varepsilon}$ is an approximation of $T$. We are thus led to study a regularization process for quasi-psh functions. In this context, we prove the following result.

TheOREM 1.1. - Let $T$ be a closed almost positive $(1,1)$-current and let $\alpha$ be a smooth real $(1,1)$-form in the same $\partial \bar{\partial}$-cohomology class as $T$, i.e. $T=\alpha+\frac{i}{\pi} \partial \bar{\partial} \psi$ where $\psi$ is an almost psh function. Let $\gamma$ be a continuous real $(1,1)$-form such that $T \geq \gamma$. Suppose that $T_{X}$ is equipped with a smooth hermitian metric $\omega$ such that the Chern curvature form $\Theta\left(T_{X}\right)$ satisfies

$$
\left(\Theta\left(T_{X}\right)+u \otimes \operatorname{Id}_{T_{X}}\right)(\theta \otimes \xi, \theta \otimes \xi) \geq 0 \quad \forall \theta, \xi \in T_{X} \quad \text { with }\langle\theta, \xi\rangle=0,
$$

for some continuous nonnegative $(1,1)$-form $u$ on $X$. Then there is a family of closed almost positive $(1,1)$-currents $\left.T_{\varepsilon}=\alpha+\frac{i}{\pi} \partial \bar{\partial} \psi_{\varepsilon}, \varepsilon \in\right] 0, \varepsilon_{0}\left[\right.$, such that $\psi_{\varepsilon}$ is smooth over $X$, increases with $\varepsilon$, and converges to $\psi$ as $\varepsilon$ tends to 0 (in particular, $T_{\varepsilon}$ is smooth and converges weakly to $T$ on $\left.X\right)$, and such that 
(i) $T_{\varepsilon} \geq \gamma-\lambda_{\varepsilon} u-\delta_{\varepsilon} \omega$ where:

(ii) $\lambda_{\varepsilon}(x)$ is an increasing family of continuous functions on $X$ such that $\lim _{\varepsilon \rightarrow 0} \lambda_{\varepsilon}(x)=\nu(T, x)$ (Lelong number of $T$ at $x$ ) at every point,

(iii) $\delta_{\varepsilon}$ is an increasing family of positive constants such that $\lim _{\varepsilon \rightarrow 0} \delta_{\varepsilon}=0$.

More precise results are given in Theorems 4.1 and 6.1. Such approximations can in turn be used to obtain various estimates of intersection theory [De91b], or asymptotic inequalities for Dolbeault cohomology [De90]. They can be also applied to study compact complex manifolds with partially semipositive curvature in the sense of Griffiths (see Section 5); in that case, we prove for instance that every effective divisor is nef (i.e. numerically effective), and that the variety is projective if and only if it is Moishezon.

Our proof uses some ideas already developed in [De82], although more general and more precise results will be obtained here. The main idea is to use a convolution kernel constructed by means of the exponential map associated to a Chern connection on $T_{X}$. To get precise estimates of the Hessian forms involved, we determine the Taylor expansion of the exponential map at order 3 . The third order coefficients can be calculated explicitly in terms of the curvature tensor of the metric. What is perhaps most remarkable is that we have been ultimately able to find the complete Taylor expansion of the exponential convolution kernel (Proposition 3.8); this is indeed possible because we use a modified exponential map which is made fiberwise quasi-holomorphic (see Section 2). Finally, we apply Kiselman's singularity attenuation technique [Ki78], [Ki79] in combination with our main estimates to define a partial regularization process for closed $(1,1)$-currents: in that way, the Lelong numbers can be killed up to any given level (Theorem 6.1).

Further techniques based on Hörmander's $L^{2}$ existence theorems [Hö66] are explained in our recent papers [De91a], [De92]; they lead to similar estimates, but with a numerical hypothesis instead of a curvature hypothesis: namely, $u$ should then be a closed real $(1,1)$-form such that the cohomology class $c_{1}\left(\mathcal{O}_{T_{X}}(1)\right)+\pi^{\star} u$ is nef on the total space of the projective bundle $P\left(T_{X}^{\star}\right) \stackrel{\pi}{\rightarrow} X$ of hyperplanes in $T_{X}$. This condition, which is more natural than a curvature hypothesis from the point of view of algebraic geometry, is also more general than the Griffiths semipositivity of $\Theta\left(T_{X}\right)+u \otimes \operatorname{Id}_{T_{X}}$. However, it is not clear how the above numerical condition can be related to the partial semipositivity hypothesis made in Theorem 1.1 (see the comments after Definition 5.1); for instance, the partial semipositivity hypothesis is void for curves. Therefore, both types of hypotheses seem to have their own domain of applicability. Moreover, the techniques developed here are considerably simpler and in some sense more precise and more explicit, so we felt interesting to explain this simpler method, which is probably easier to extend to currents of higher bidegrees. The main ideas of this work have been worked out during a stay of the author at Bayreuth University in November 1989. The author wishes to thank this Institution for its hospitality. 


\section{Exponential map associated to the Chern connection}

Suppose that the manifold $X$ is equipped with a smooth hermitian metric $\omega=i \sum \omega_{l m} d z_{l} \wedge d \bar{z}_{m}$. Denote by $D$ the Chern connection of $T_{X}$ and by $\Theta\left(T_{X}\right)=$ $\frac{i}{2 \pi} D^{2}$ the curvature tensor. We define an exponential map exp $: T_{X} \longrightarrow X$ as follows: if $\zeta \in T_{X, z}$, then $\exp _{z}(\zeta)$ is the position at time $t=1$ of the curve $t \mapsto u(t)$ starting at $u(0)=z$ with initial tangent vector $u^{\prime}(0)=\zeta$ and satisfying the second order differential equation $D(d u / d t)=0$ (parallel translation with respect to $D$ ). If $\omega$ is Kähler, the Chern connection coincides with the Levi-Civita connection, so exp is given in that case by the riemannian geodesics; otherwise, exp differs from the usual riemannian exponential map. For any $x \in X$, fix analytic coordinates $\left(z_{1}, \ldots, z_{n}\right)$ centered at $x$ such that $\left(\partial / \partial z_{l}\right)$ is an orthonormal basis of $T_{X}$ at $x$. Consider the Taylor expansion of second order

$$
\begin{aligned}
\omega_{l m}(z)=\left\langle\frac{\partial}{\partial z_{l}}, \frac{\partial}{\partial z_{m}}\right\rangle=\delta_{l m}+\sum_{j}\left(a_{j l m} z_{j}+\bar{a}_{j m l} \bar{z}_{j}\right) & +\sum_{j, k}\left(b_{j k l m}^{\prime} z_{j} z_{k}+\bar{b}_{j k m l}^{\prime} \bar{z}_{j} \bar{z}_{k}\right) \\
& +\sum_{j, k} c_{j k l m}^{\prime} z_{j} \bar{z}_{k}+O\left(|z|^{3}\right) .
\end{aligned}
$$

We may always arrange that the antisymmetry relation $a_{j l m}=-a_{l j m}$ holds; otherwise the change of variables $z_{m}=z_{m}^{\prime}-\frac{1}{4} \sum\left(a_{j l m}+a_{l j m}\right) z_{j}^{\prime} z_{l}^{\prime}$ yields coordinates $\left(z_{l}^{\prime}\right)$ with this property. If $\omega$ is Kähler, the symmetry of $a_{j l m}=\partial \omega_{l m} / \partial z_{j}$ in $j, l$ implies $a_{j l m}=0$; in that case $b_{j k l m}^{\prime}$ is also symmetric in $j, k, l$ and a new change of variables $z_{m}=z_{m}^{\prime}-\frac{1}{3} \sum b_{j k l m}^{\prime} z_{j}^{\prime} z_{k}^{\prime} z_{l}^{\prime}$ gives $b_{j k l m}^{\prime}=0$ likewise. The holomorphic frame of $T_{X}$ defined by

satisfies

$$
e_{l}=\frac{\partial}{\partial z_{l}}-\sum_{m}\left(\sum_{j} a_{j l m} z_{j}+\sum_{j, k} b_{j k l m}^{\prime} z_{j} z_{k}\right) \frac{\partial}{\partial z_{m}}
$$

$$
\begin{aligned}
\left\langle e_{l}, e_{m}\right\rangle & =\delta_{l m}-\sum_{j, k} c_{j k l m} z_{j} \bar{z}_{k}+O\left(|z|^{3}\right), \\
\frac{\partial}{\partial z_{l}} & =e_{l}+\sum_{m}\left(\sum_{j} a_{j l m} z_{j}+\sum_{j, k} b_{j k l m} z_{j} z_{k}+O\left(z^{3}\right)\right) e_{m}
\end{aligned}
$$

with $c_{j k l m}=-c_{j k l m}^{\prime}-\sum_{p} a_{j l p} \bar{a}_{k m p}$ and $b_{j k l m}=b_{j k l m}^{\prime}+\sum_{p} a_{j l p} a_{k p m}$. We may of course suppose that $b_{j k l m}=b_{k j l m}$. Also, by a modification of the third order terms in $\left(e_{l}\right)$, we can suppose that no term $O\left(z^{3}\right)$ appears in (2.2). The formula $\partial\left\langle e_{l}, e_{m}\right\rangle=\left\langle D e_{l}, e_{m}\right\rangle$ easily gives the expression of $D e_{l}, D^{2} e_{l}$ and $\Theta\left(T_{X}\right)_{x}$ :

$$
\begin{aligned}
D e_{l} & =-\sum_{j, k, m} c_{j k l m} \bar{z}_{k} d z_{j} \otimes e_{m}+O\left(|z|^{2}\right), \\
\Theta\left(T_{X}\right)_{x} & =\frac{i}{2 \pi} \sum_{j, k, l, m} c_{j k l m} d z_{j} \wedge d \bar{z}_{k} \otimes e_{l}^{\star} \otimes e_{m} .
\end{aligned}
$$

Given a vector field $\zeta=\sum \zeta_{l} \partial / \partial z_{l}$ in $T_{X}$, we denote by $\left(\xi_{l}\right)$ the components of $\zeta$ with respect to the basis $\left(e_{l}\right)$, thus $\zeta=\sum \xi_{l} e_{l}$. By (2.2) we have

$$
\xi_{m}=\zeta_{m}+\sum_{j, l} a_{j l m} z_{j} \zeta_{l}+\sum_{j, k, l} b_{j k l m} z_{j} z_{k} \zeta_{l} \text {. }
$$


In the Kähler case everything is much simpler, we take $e_{l}=\partial / \partial z_{l}$ and $\xi_{m}=\zeta_{m}$. In general, the Chern connection $D$ is given by $D \zeta=D\left(\sum \zeta_{l} \partial / \partial z_{l}\right)$ with

$$
\begin{aligned}
D\left(\frac{\partial}{\partial z_{l}}\right)= & -\sum_{j, k, m} c_{j k l m} \bar{z}_{k} d z_{j} \otimes e_{m} \\
& +\sum_{j, m} a_{j l m} d z_{j} \otimes e_{m} \\
& +2 \sum_{j, k, m} b_{j k l m} z_{k} d z_{j} \otimes e_{m}+O\left(|z|^{2}\right) d z \\
= & -\sum_{j, k, m}\left(c_{j k l m} \bar{z}_{k}-2 b_{j k l m} z_{k}\right) d z_{j} \otimes \frac{\partial}{\partial z_{m}} \\
& +\sum_{j, m}\left(a_{j l m}-\sum_{k, p} a_{j l p} a_{k p m} z_{k}\right) d z_{j} \otimes \frac{\partial}{\partial z_{m}}+O\left(|z|^{2}\right) d z, \\
D \zeta= & \sum_{m} d \zeta_{m} \otimes \frac{\partial}{\partial z_{m}}-\sum_{j, k, l, m}\left(c_{j k l m} \bar{z}_{k}-2 b_{j k l m} z_{k}\right) \zeta_{l} d z_{j} \otimes \frac{\partial}{\partial z_{m}} \\
& +\sum_{j, l, m}\left(a_{j l m}-\sum_{k, p} a_{j l p} a_{k p m} z_{k}\right) \zeta_{l} d z_{j} \otimes \frac{\partial}{\partial z_{m}}+O\left(|z|^{2}\right) \cdot \zeta d z .
\end{aligned}
$$

Consider a curve $t \mapsto u(t)$. By a substitution of variables $z_{j}=u_{j}(t), \zeta_{l}=d u_{l} / d t$ in formula $(2.5)$, the equation $D(d u / d t)=0$ becomes

$$
\frac{d^{2} u_{m}}{d t^{2}}=\sum_{j, k, l}\left(c_{j k l m} \bar{u}_{k}(t)-2 b_{j k l m} u_{k}(t)\right) \frac{d u_{j}}{d t} \frac{d u_{l}}{d t}+O\left(|u(t)|^{2}\right) \cdot\left(\frac{d u}{d t}\right)^{2} ;
$$

the contribution of the terms $\sum a_{j l} \zeta_{l} d z_{j}$ is zero by the antisymmetry relation; moreover the remainder term only contains $\mathbb{C}$-quadratic terms in $d u / d t$. The initial condition $u(0)=z, u^{\prime}(0)=\zeta$ gives $u_{m}(t)=z_{m}+t \zeta_{m}+O\left(t^{2}|\zeta|^{2}\right)$, hence

$$
\frac{d^{2} u_{m}}{d t^{2}}=\sum_{j, k, l}\left(c_{j k l m}\left(\bar{z}_{k}+t \bar{\zeta}_{k}\right)-2 b_{j k l m}\left(z_{k}+t \zeta_{k}\right)\right) \zeta_{j} \zeta_{l}+O\left(|\zeta|^{2}(|z|+|\zeta|)^{2}\right) \text {. }
$$

Two successive integrations yield

$$
\begin{aligned}
u_{m}(t)=z_{m}+t \zeta_{m} & +\sum_{j, k, l} c_{j k l m}\left(\frac{t^{2}}{2} \bar{z}_{k}+\frac{t^{3}}{6} \bar{\zeta}_{k}\right) \zeta_{j} \zeta_{l} \\
& -2 \sum_{j, k, l} b_{j k l m}\left(\frac{t^{2}}{2} z_{k}+\frac{t^{3}}{6} \zeta_{k}\right) \zeta_{j} \zeta_{l}+O\left(t^{2}|\zeta|^{2}(|z|+|\zeta|)^{2}\right) .
\end{aligned}
$$

An iteration of this procedure (substitution in (2.6) followed by an integration) easily shows that all terms but the first two in the Taylor expansion of $u_{m}(t)$ contain $\mathbb{C}$-quadratic factors of the form $\zeta_{j} \zeta_{l}$. Let us substitute $\zeta_{m}$ by its expression in terms of $z, \xi$ deduced from (2.4). We find that $\exp _{z}(\zeta)=u(1)$ has a third order expansion

$$
\exp _{z}(\zeta)_{m}=g_{m}(z, \xi)+\sum_{j, k, l} c_{j k l m}\left(\frac{1}{2} \bar{z}_{k}+\frac{1}{6} \bar{\xi}_{k}\right) \xi_{j} \xi_{l}+O\left(|\xi|^{2}(|z|+|\xi|)^{2}\right)
$$


where

$$
\begin{aligned}
g_{m}(z, \xi)=z_{m}+\xi_{m} & -\sum_{j, l} a_{j l m} z_{j} \xi_{l}+\sum_{j, k, l, p} a_{j l p} a_{k p m} z_{j} z_{k} \xi_{l} \\
& -\sum_{j, k, l} b_{j k l m}\left(z_{j} z_{k} \xi_{l}+z_{k} \xi_{j} \xi_{l}+\frac{1}{3} \xi_{j} \xi_{k} \xi_{l}\right)
\end{aligned}
$$

is a holomorphic polynomial of degree 3 in $z, \xi$ and where the remainder involves $\mathbb{C}$-quadratic factors $\xi_{j} \xi_{l}$ in all terms. In the Kähler case we simply have $\xi_{m}=\zeta_{m}$ and $g_{m}(z, \xi)=z_{m}+\xi_{m}$.

The exponential map is unfortunately non holomorphic. However, we can make it quasi-holomorphic with respect to $\zeta$ as follows: for $z$ fixed, we consider the formal power series obtained by eliminating all monomials in the Taylor expansion of $\zeta \mapsto \exp _{z}(\zeta)$ at the origin which are not holomorphic with respect to $\zeta$. This defines in a unique way a jet of infinite order along the zero section of $T_{X}$. E. Borel's theorem shows that there is a smooth map

$$
T_{X} \longrightarrow X, \quad(z, \zeta) \longmapsto \operatorname{exph}_{z}(\zeta)
$$

such that its jet at $\zeta=0$ coincides with the "holomorphic" part of $\zeta \mapsto \exp _{z}(\zeta)$ (of course, this map is defined only up to an addition of flat $C^{\infty}$ functions along the zero section of $T_{X}$ ). Moreover, (2.7) implies that

$$
\operatorname{exph}_{z}(\zeta)_{m}=g_{m}(z, \xi)+\frac{1}{2} \sum_{j, k, l} c_{j k l m} \bar{z}_{k} \xi_{j} \xi_{l}+O\left(|\xi|^{2}(|z|+|\xi|)^{2}\right)
$$

By including in $g_{m}$ all holomorphic monomials of partial degree at most 2 in $z$ and $N$ in $\xi\left(N \geq 2\right.$ being a given integer), we get holomorphic polynomials $h_{m}(z, \xi)$ of linear part $z_{m}+\xi_{m}$ and total degree $N+2$, such that

$$
\operatorname{exph}_{z}(\zeta)_{m}=h_{m}(z, \xi)+O\left(\bar{z}, z \bar{z}, \overline{z z},|z|^{3}, \xi^{N-1}\right) \xi^{2} .
$$

Here a notation as $O\left(\bar{z}, z \bar{z}, \overline{z z},|z|^{3}, \xi^{N-1}\right) \xi^{2}$ indicates an arbitrary function in the ideal of $C^{\infty}$ functions generated by monomials of the form $\bar{z}_{k} \xi_{l} \xi_{m}, z_{i} \bar{z}_{j} \xi_{l} \xi_{m}$, $\bar{z}_{i} \bar{z}_{j} \xi_{l} \xi_{m}, z^{\alpha} \bar{z}^{\beta} \xi_{l} \xi_{m}$ and $\xi^{\gamma}$, for all multi-indices $|\alpha|+|\beta|=3$ and $|\gamma|=N+1$ (the notation $|z|^{3}$ thus stands for an arbitrary monomial of degree 3 in $z$, $\bar{z}$, so that $O\left(|z|^{3}\right)$ is compatible with the usual Landau notation). By the implicit function theorem applied to the mapping $h=\left(h_{m}\right)_{1 \leq m \leq n}$ we thus get:

Proposition 2.9. - Let $\omega$ be a smooth hermitian metric on $X$. There exists a $C^{\infty}$ map

$$
T_{X} \longrightarrow X, \quad(x, \zeta) \longmapsto \operatorname{exph}_{x}(\zeta), \quad \zeta \in T_{X, x}
$$

with the following properties:

(i) For every $x \in X$, $\operatorname{exph}_{x}(0)=x$ and $d_{\zeta} \operatorname{exph}_{x}(0)=\operatorname{Id}_{T_{X, x}}$.

(ii) For every $x \in X$, the $\operatorname{map} \zeta \mapsto \operatorname{exph}_{x}(\zeta)$ has a holomorphic Taylor expansion at $\zeta=0$. Moreover, with respect to $\omega$, there are local normal coordinates 
$\left(z_{1}, \ldots, z_{n}\right)$ on $X$ centered at $x$ and holomorphic normal coordinates $\left(\xi_{j}\right)$ on the fibers of $T_{X}$ near $x$ such that

$$
\operatorname{exph}_{z}(\zeta)=h_{x}\left(z, \rho_{x}(z, \xi)\right)
$$

where $h_{x}(z, \xi)$ is a holomorphic polynomial map of degree 2 in $z$ and of degree $N$ in $\xi$, and where $\rho_{x}: \mathbb{C}^{n} \times \mathbb{C}^{n} \rightarrow \mathbb{C}^{n}$ is a smooth map such that

$$
\begin{gathered}
h_{x, m}(z, \xi)=z_{m}+\xi_{m}-\sum_{j, l} a_{j l m} z_{j} \xi_{l}+\sum_{j, k, l, p} a_{j l p} a_{k p m} z_{j} z_{k} \xi_{l} \\
-\sum_{j, k, l} b_{j k l m}\left(z_{j} z_{k} \xi_{l}+z_{k} \xi_{j} \xi_{l}+\frac{1}{3} \xi_{j} \xi_{k} \xi_{l}\right) \\
+O\left((|z|+|\xi|)^{4}\right), \\
\rho_{x, m}(z, \xi)=\xi_{m}+\sum_{2 \leq|\alpha| \leq N}\left(\sum_{k} d_{\alpha k m} \xi^{\alpha} \bar{z}_{k}+\sum_{j, k} e_{\alpha j k m} \xi^{\alpha} z_{j} \bar{z}_{k}\right) \\
+O\left(\bar{z}^{2},|z|^{3}, \xi^{N-1}\right) \xi^{2} .
\end{gathered}
$$

(iii) For $\alpha=\left(0, \ldots, 1_{j}, \ldots, 1_{l}, \ldots, 0\right)$ of degree 2 , we have $d_{\alpha k m}=\frac{1}{2} c_{j k l m}$ where $\left(c_{j k l m}\right)$ is the curvature tensor of $\omega$ at $x$.

Of course, if the hermitian metric $\omega$ is real analytic, all the above expansions are convergent, hence $\operatorname{exph}_{z}(\zeta)$ is real analytic and holomorphic in $\zeta$ in a neighborhood of the zero section of $T_{X}$. By taking $N=\infty$, we obtain a real analytic map $\rho(x, \xi)$ which is holomorphic in $\xi$, so the above remainder term becomes $O\left(\bar{z}^{2},|z|^{3}\right) \xi^{2}$.

\section{Regularization of quasi-psh functions}

We now come to the main idea. Select a cut-off function $\chi: \mathbb{R} \rightarrow \mathbb{R}$ of class $C^{\infty}$ such that

$$
\chi(t)>0 \text { for } t<1, \quad \chi(t)=0 \text { for } t \geq 1, \quad \int_{v \in \mathbb{C}^{n}} \chi\left(|v|^{2}\right) d \lambda(v)=1 .
$$

If $\psi$ is a quasi-psh function on $X$, we set

$$
\psi_{\varepsilon}(z)=\frac{1}{\varepsilon^{2 n}} \int_{\zeta \in T_{X, z}} \psi\left(\operatorname{exph}_{z}(\zeta)\right) \chi\left(\frac{|\zeta|^{2}}{\varepsilon^{2}}\right) d \lambda(\zeta), \quad \varepsilon>0 .
$$

Here $d \lambda$ denotes the Lebesgue measure on $\mathbb{C}^{n}$, resp. on the hermitian space $\left(T_{X, z}, \omega(z)\right)$. For $w \in \mathbb{C}$ with $|w|=\varepsilon$, we have $\psi_{\varepsilon}(z)=\Psi(z, w)$ with

$$
\Psi(z, w)=\int_{\zeta \in T_{X, z}} \psi\left(\operatorname{exph}_{z}(w \zeta)\right) \chi\left(|\zeta|^{2}\right) d \lambda(\zeta) .
$$

The change of variable $y=\operatorname{exph}_{z}(w \zeta)$ expresses $w \zeta$ as a smooth function of $y, z$ in a neighborhood of the diagonal in $X \times X$. Hence $\Psi$ is smooth over $X \times\left\{0<|w|<\varepsilon_{0}\right\}$ for some $\varepsilon_{0}>0$. We are going to compute $\partial \bar{\partial} \Psi$ over this set and estimate its 
negative part when $|w|$ is small. For this, we fix a point $x \in X$ and use the coordinates $(z, \xi)$ on $T_{X}$ introduced in $\S 2$; for simplicity, we omit the index $x$ in the notation of $h_{x}$ and $\rho_{x}$. By (2.1), we have

$$
\begin{aligned}
|\zeta|^{2} & =\sum_{m}\left|\xi_{m}\right|^{2}-\sum_{j, k, l, m} c_{j k l m} z_{j} \bar{z}_{k} \xi_{l} \bar{\xi}_{m}+O\left(|z|^{3}\right)|\xi|^{2} \\
d \lambda(\zeta) & =\frac{1}{2^{n} n !}\left(i \partial \bar{\partial}|\zeta|^{2}\right)^{n} \\
& =\left(1-\sum_{j, k, l} c_{j k l l} z_{j} \bar{z}_{k}+O\left(|z|^{3}\right)\right) \frac{i}{2} d \xi_{1} \wedge d \bar{\xi}_{1} \wedge \ldots \wedge \frac{i}{2} d \xi_{n} \wedge d \bar{\xi}_{n}
\end{aligned}
$$

In (3.2), we make the change of variables $s=w^{-1} \rho(z, w \xi)$, hence we can write $\operatorname{exph}_{z}(w \zeta)=h(z, w s)$. By (2.9) we get

$$
\begin{aligned}
s_{m}=\xi_{m}+\sum_{2 \leq|\alpha| \leq N}\left(\sum_{k} d_{\alpha k m} w^{|\alpha|-1} \xi^{\alpha} \bar{z}_{k}\right. & \left.+\sum_{j, k} e_{\alpha j k m} w^{|\alpha|-1} \xi^{\alpha} z_{j} \bar{z}_{k}\right) \\
& +O\left(\bar{z}^{2},|z|^{3}, w^{N-1} \xi^{N-1}\right) w \xi^{2}
\end{aligned}
$$

Therefore

$$
\begin{aligned}
\xi_{m}=s_{m}-\sum_{2 \leq|\alpha| \leq N}\left(\sum_{k} d_{\alpha k m} w^{|\alpha|-1} s^{\alpha} \bar{z}_{k}\right. & \left.+\sum_{j, k} e_{\alpha j k m} w^{|\alpha|-1} s^{\alpha} z_{j} \bar{z}_{k}\right) \\
& +O\left(\bar{z}^{2},|z|^{3}, w^{N-1} s^{N-1}\right) w s^{2}
\end{aligned}
$$

and $\xi=s+O\left(w^{N} s^{N+1}\right)$ for $z=0$. After a substitution in (3.2), (3.3), (3.4) we get

$$
\Psi(z, w)=\int_{\mathbb{C}^{n}} \psi(h(z, w s)) \chi(A(z, w, s)) B(z, w, s) d \lambda(s)
$$

where

$$
\begin{aligned}
& A(z, w, s)=\sum_{m}\left|s_{m}\right|^{2}-\sum_{j, k, l, m} c_{j k l m} z_{j} \bar{z}_{k} s_{l} \bar{s}_{m} \\
&-2 \operatorname{Re} \sum_{\alpha, k, m} d_{\alpha k m} w^{|\alpha|-1} s^{\alpha} \bar{s}_{m} \bar{z}_{k} \\
&-2 \operatorname{Re} \sum_{\alpha, j, k, m} e_{\alpha j k m} w^{|\alpha|-1} s^{\alpha} \bar{s}_{m} z_{j} \bar{z}_{k} \\
&+\sum_{\alpha, \beta, j, k, m} d_{\alpha k m} \overline{d_{\beta j m}} w^{|\alpha|-1} \bar{w}^{|\beta|-1} s^{\alpha} \bar{s}^{\beta} z_{j} \bar{z}_{k} \\
&+ O\left(z^{2}, \bar{z}^{2},|z|^{3},|w|^{N-1}|s|^{N-1}\right)|w||s|^{3},
\end{aligned}
$$




$$
\begin{aligned}
B(z, w, s)=1 & -\sum_{j, k, l} c_{j k l l} z_{j} \bar{z}_{k} \\
& -2 \operatorname{Re} \sum_{\alpha, k, m} d_{\alpha k m} w^{|\alpha|-1} \alpha_{m} s^{\alpha-\mathbf{1}_{m}} \bar{z}_{k} \\
& -2 \operatorname{Re} \sum_{\alpha, j, k, m} e_{\alpha j k m} w^{|\alpha|-1} \alpha_{m} s^{\alpha-\mathbf{1}_{m}} z_{j} \bar{z}_{k} \\
& +\sum_{\alpha, \beta, j, k, l, m} d_{\alpha k m} \overline{d_{\beta j l}} w^{|\alpha|-1} \bar{w}^{|\beta|-1} \alpha_{m} \beta_{l} s^{\alpha-\mathbf{1}_{m}} \bar{s}^{\beta-\mathbf{1}_{l}} z_{j} \bar{z}_{k} \\
& +O\left(z^{2}, \bar{z}^{2},|z|^{3},|w|^{N-1}|s|^{N-1}\right)|w||s| ;
\end{aligned}
$$

here $\left(\mathbf{1}_{m}\right)_{1 \leq m \leq n}$ denotes the standard basis of $\mathbb{Z}^{n}$, hence $s^{\mathbf{1}_{m}}=s_{m}$.

Proposition 3.8. - For any integer $N \geq 2$ and any $(\theta, \eta) \in T_{X, x} \times \mathbb{C}$, the Hessian form of $\Psi$ at $(x, w) \in X \times \mathbb{C}$ satisfies an estimate

$$
\begin{gathered}
\partial \bar{\partial} \Psi_{(x, w)}[\theta, \eta]^{2}=\int_{\zeta \in T_{X, x}} \partial \bar{\partial} \psi \cdot(\tau \wedge \bar{\tau} \\
\left.+|w|^{2} V\right)_{\operatorname{exph}_{x}(w \zeta)} \chi\left(|\zeta|^{2}\right) d \lambda(\zeta) \\
+O\left(|w|^{N-1}\right)[\theta, \eta]^{2}
\end{gathered}
$$

where $\tau$ (resp. $V$ ) is a vector field (resp. a $(1,1)$-vector field) depending smoothly on the parameters $x, w$ and linearly (resp. quadratically) on $\theta, \eta$, and where the notation $[\tau]^{2}$ stands for the $(1,1)$-vector $\tau \wedge \bar{\tau} \in \Lambda^{1,1} T_{X}$. The vector fields $\tau, V$ are given at $y=\operatorname{exph}_{x}(w \zeta)$ by

$$
\begin{aligned}
\tau_{y} & =\partial \operatorname{exph}_{(x, w \zeta)}\left(\theta^{h}+\eta \zeta^{v}+|w|^{2} \Xi_{y}^{v}\right), \\
V_{y} & =\partial \operatorname{exph}_{(x, w \zeta)}\left(U^{v}-|w|^{2} \Xi^{v} \wedge \bar{\Xi}^{v}\right)_{y},
\end{aligned}
$$

where $\theta^{h}, \zeta^{v} \in T\left(T_{X}\right)_{(x, w \zeta)}$ are respectively the horizontal lifting of $\theta$ with respect to the connection $D$ and the vertical tangent vector associated to $\zeta$, and where $\Xi, U$ can be expressed in the coordinates 2.9 (ii) by

$$
\begin{aligned}
\Xi_{y}= & \sum_{\alpha, j, l, m} \frac{1}{\chi\left(|\zeta|^{2}\right)} \frac{\partial}{\partial \bar{\zeta}_{l}}\left(\chi_{1}\left(|\zeta|^{2}\right) \bar{\zeta}^{\alpha-\mathbf{1}_{m}}\right) \overline{d_{\alpha j l}} \frac{\alpha_{m}}{|\alpha|} \bar{w}^{|\alpha|-2} \theta_{j} \frac{\partial}{\partial z_{m}} \\
U_{y}= & \sum_{l, m} \frac{1}{2}\left(U_{m, l}+\overline{U_{l, m}}\right) \frac{\partial}{\partial z_{m}} \wedge \frac{\partial}{\partial \bar{z}_{l}}, \\
U_{m, l}= & -\frac{\chi_{1}\left(|\zeta|^{2}\right)}{\chi\left(|\zeta|^{2}\right)}\left\{\sum_{j, k} c_{j k l m} \theta_{j} \bar{\theta}_{k}\right. \\
& +2 \sum_{\alpha, j, k} e_{\alpha j k m} w^{|\alpha|-1} \frac{\alpha_{l}}{|\alpha|} \zeta^{\alpha-\mathbf{1}_{l}} \theta_{j} \bar{\theta}_{k} \\
& \left.+2 \sum_{\alpha, k} d_{\alpha k m}(|\alpha|-1) w^{|\alpha|-2} \frac{\alpha_{l}}{|\alpha|} \zeta^{\alpha-\mathbf{1}_{l}} \eta \bar{\theta}_{k}\right\} \\
& +\sum_{\alpha, \beta, j, k} d_{\alpha k m} \frac{d_{\beta j l}}{w^{|\alpha|-1} \bar{w}^{|\beta|-1} \zeta^{\alpha} \bar{\zeta}^{\beta} \theta_{j} \bar{\theta}_{k} .}
\end{aligned}
$$


Here $\left(c_{j k l m}\right)$ is the curvature tensor of the given hermitian metric $\omega$ on $X$, and $\chi_{1}$ denotes the primitive $\chi_{1}(t)=\int_{+\infty}^{t} \chi(u) d u$ of $\chi$ such that $\chi_{1}(t)=0$ for $t \geq 1$. Moreover, $\alpha, \beta \in \mathbb{N}^{n}$ run over all multi-indices such that $2 \leq|\alpha|,|\beta| \leq N$. The formula is exact when $\omega$ is real analytic and $N=\infty$.

Proof. - A brute force differentiation of (3.7) at $z=0$ gives

$$
\begin{aligned}
\partial \bar{\partial} \Psi_{(x, w)}[\theta, \eta]^{2}= & \int_{\mathbb{C}^{n}} \partial \bar{\partial}(\psi \circ h)_{(0, w s)}[\theta, \eta s]^{2} \chi(A(0, w, s)) B(0, w, s) d \lambda(s) \\
& -\int_{\mathbb{C}^{n}} \bar{\partial}(\psi \circ h)_{(0, w s)}[\theta, \eta s] E_{(w, s)}[\theta, \eta] d \lambda(s) \\
& -\int_{\mathbb{C}^{n}} \partial(\psi \circ h)_{(0, w s)}[\theta, \eta s] \overline{E_{(w, s)}[\theta, \eta]} d \lambda(s) \\
& -\int_{\mathbb{C}^{n}} \psi \circ h(0, w s) F_{(w, s)}[\theta, \eta]^{2} d \lambda(s)
\end{aligned}
$$

where

$$
\begin{aligned}
& E_{(w, s)}=-\partial_{(z, w)}(\chi(A(z, w, s)) B(z, w, s)), \\
& F_{(w, s)}=-\partial \bar{\partial}_{(z, w)}(\chi(A(z, w, s)) B(z, w, s))
\end{aligned}
$$

at $z=0,|w|<\varepsilon_{0}$. Clearly, terms $w^{p} z \bar{z}$ in $A$ or $B$ play no role in $E_{(w, s)}$, while terms $w^{p} \bar{z}$ contribute either linearly as $d w \wedge d \bar{z}$ differentials of $F_{(w, s)}$ or quadratically as $d z \wedge d \bar{z}$ differentials. This gives

$$
\begin{aligned}
E_{(w, s)}[\theta, \eta]=\chi^{\prime} & \left(|s|^{2}\right) \sum_{\alpha, j, l} \overline{d_{\alpha j l}} \bar{w}^{|\alpha|-1} \bar{s}^{\alpha} s_{l} \theta_{j} \\
& +\chi\left(|s|^{2}\right) \sum_{\alpha, j, l} \overline{d_{\alpha j l}} \bar{w}^{|\alpha|-1} \alpha_{l} \bar{s}^{\alpha-1} \theta_{j}+O\left(|w|^{N-1}|s|^{N}\right)[\theta, \eta] \\
F_{(w, s)}[\theta, \eta]^{2}= & \chi^{\prime}\left(|s|^{2}\right) \sum_{j, k, l, m} c_{j k l m} s_{l} \bar{s}_{m} \theta_{j} \bar{\theta}_{k}+\chi\left(|s|^{2}\right) \sum_{j, k, l} c_{j k l l} \theta_{j} \bar{\theta}_{k} \\
& +2 \operatorname{Re}\left\{\chi^{\prime}\left(|s|^{2}\right) \sum_{\alpha, j, k, m} e_{\alpha j k m} w^{|\alpha|-1} s^{\alpha} \bar{s}_{m} \theta_{j} \bar{\theta}_{k}\right. \\
& +\chi\left(|s|^{2}\right) \sum_{\alpha, j, k, m} e_{\alpha j k m} w^{|\alpha|-1} \alpha_{m} s^{\alpha-1} \theta_{m} \bar{\theta}_{k} \\
& +\chi^{\prime}\left(|s|^{2}\right) \sum_{\alpha, k, m} d_{\alpha k m}(|\alpha|-1) w^{|\alpha|-2} s^{\alpha} \bar{s}_{m} \eta \bar{\theta}_{k} \\
& \left.+\chi\left(|s|^{2}\right) \sum_{\alpha, k, m} d_{\alpha j k m}(|\alpha|-1) w^{|\alpha|-2} \alpha_{m} s^{\alpha-1} \overline{1}_{m} \bar{\theta}_{k}\right\} \\
& -\chi^{\prime \prime}\left(|s|^{2}\right) \sum_{\alpha, \beta, j, k, l, m} d_{\alpha k m} \overline{d_{\beta j l}} w^{|\alpha|-1} \bar{w}^{|\beta|-1} s^{\alpha} \bar{s}^{\beta} s_{l} \bar{s}_{m} \theta_{j} \bar{\theta}_{k} \\
& -\chi^{\prime}\left(|s|^{2}\right) \sum_{\alpha, \beta, j, k, m} d_{\alpha k m} \overline{d_{\beta j m}} w^{|\alpha|-1} \bar{w}^{|\beta|-1} s^{\alpha} \bar{s}^{\beta} \theta_{j} \bar{\theta}_{k}
\end{aligned}
$$




$$
\begin{aligned}
& -\chi^{\prime}\left(|s|^{2}\right) \sum_{\alpha, \beta, j, k, l, m} d_{\alpha k m} \overline{d_{\beta j l}} w^{|\alpha|-1} \bar{w}^{|\beta|-1} s^{\alpha} \bar{s}_{m} \beta_{l} \bar{s}^{\beta-\mathbf{1}_{l}} \theta_{j} \bar{\theta}_{k} \\
& -\chi^{\prime}\left(|s|^{2}\right) \sum_{\alpha, \beta, j, k, l, m} d_{\alpha k m} \overline{d_{\beta j l}} w^{|\alpha|-1} \bar{w}^{|\beta|-1} \alpha_{m} s^{\alpha-\mathbf{1}_{m}} \bar{s}^{\beta} s_{l} \theta_{j} \bar{\theta}_{k} \\
& -\chi\left(|s|^{2}\right) \sum_{\alpha, \beta, j, k, l, m} d_{\alpha k m} \overline{d_{\beta j l}} w^{|\alpha|-1} \bar{w}^{|\beta|-1} \alpha_{m} \beta_{l} s^{\alpha-\mathbf{1}_{m}} \bar{s}^{\beta-\mathbf{1}_{l}} \theta_{j} \bar{\theta}_{k} \\
& +O\left(|w|^{N-2}|s|^{N}\right)[\theta, \eta]^{2} .
\end{aligned}
$$

We find

$$
\begin{aligned}
E_{(w, s)}[\theta, \eta] & =\sum_{l, m} \frac{\partial^{2}}{\partial \bar{s}_{l} \partial s_{m}}\left(\chi_{1}\left(|s|^{2}\right) \sum_{\alpha, j} \overline{d_{\alpha j l}} \bar{w}^{|\alpha|-1} \frac{\alpha_{m}}{|\alpha|} \bar{s}^{\alpha-\mathbf{1}_{m}} \theta_{j}\right) \\
& +O\left(|w|^{N-1}|s|^{N}\right)[\theta, \eta], \\
F_{(w, s)}[\theta, \eta]^{2}=\sum_{l, m} & \frac{\partial^{2}}{\partial \bar{s}_{l} \partial s_{m}}\left(\chi_{1}\left(|s|^{2}\right) \sum_{j, k} c_{j k l m} \theta_{j} \bar{\theta}_{k}\right) \\
& +2 \operatorname{Re}\left\{\sum_{l, m} \frac{\partial^{2}}{\partial \bar{s}_{l} \partial s_{m}}\left(\chi_{1}\left(|s|^{2}\right) \sum_{\alpha, j, k} e_{\alpha j k m} w^{|\alpha|-1} \frac{\alpha_{l}}{|\alpha|} s^{\alpha-\mathbf{1}_{l}} \theta_{j} \bar{\theta}_{k}\right)\right. \\
& \left.+\sum_{l, m} \frac{\partial^{2}}{\partial s_{l} \partial \bar{s}_{m}}\left(\chi_{1}\left(|s|^{2}\right) \sum_{\alpha, k} d_{\alpha k m}(|\alpha|-1) w^{|\alpha|-2} \frac{\alpha_{l}}{|\alpha|} \bar{s}^{\alpha-\mathbf{1}_{l}} \eta \bar{\theta}_{k}\right)\right\} \\
& -\sum_{l, m} \frac{\partial^{2}}{\partial \bar{s}_{l} \partial s_{m}}\left(\chi\left(|s|^{2}\right) \sum_{\alpha, \beta, j, k} d_{\alpha k m} \overline{d_{\beta j l}} w^{|\alpha|-1} \bar{w}^{|\beta|-1} s^{\alpha} \bar{s}^{\beta} \theta_{j} \bar{\theta}_{k}\right) \\
& +O\left(|w|^{N-2}|s|^{N}\right)[\theta, \eta]^{2} .
\end{aligned}
$$

In all these expansions, the remainder terms $O(\bullet)$ involve uniform constants when the origin $x$ of coordinates belongs to a compact subset of a coordinate patch. By the mean value properties of plurisubharmonic functions, we have

$$
\int_{|s|<1}|\psi(x+w s)| d \lambda(s) \leq C(1+|\log | w||)
$$

locally uniformly in $x$. An integration by parts with compact supports yields

$$
\int_{|s|<1} \partial(\psi \circ h)_{(0, w s)} O(|w|) d \lambda(s)=\int_{|s|<1} \psi \circ h(0, w s) d \lambda(s)=O(\log |w|),
$$

hence the remainder terms $O\left(|w|^{N-1}\right)$ in $E_{(w, s)}$ and $O\left(|w|^{N-2}\right)$ in $F_{(w, s)}$ give contributions of order at most $O\left(|w|^{N-2} \log |w|\right)$ in $\partial \bar{\partial} \Psi$ as $|w|$ tends to 0 . An integration by parts in $(3.9)$ and $\left(3.9^{\prime}\right)$ gives

$$
\begin{aligned}
\partial \bar{\partial} \Psi_{(x, w)} & {[\theta, \eta]^{2}=\int_{\mathbb{C}^{n}} \partial \bar{\partial}(\psi \circ h)_{(0, w s)} \cdot\left\{(\theta, \eta s) \wedge \overline{(\theta, \eta s)}+|w|^{2}(0, \Xi) \wedge \overline{(\theta, \eta s)}\right.} \\
& \left.+|w|^{2}(\theta, \eta s) \wedge \overline{(0, \Xi)}+|w|^{2}(0, U)\right\} \chi(A(0, w, s)) B(0, w, s) d \lambda(s) \\
& +O\left(|w|^{N-2} \log |w|\right)[\theta, \eta]^{2}
\end{aligned}
$$


with

$$
\begin{aligned}
& \Xi=\sum_{\alpha, j, l, m} \frac{1}{\chi\left(|s|^{2}\right)} \frac{\partial}{\partial \bar{s}_{l}}\left(\chi_{1}\left(|s|^{2}\right) \bar{s}^{\alpha-\mathbf{1}_{m}}\right) \overline{d_{\alpha j l}} \frac{\alpha_{m}}{|\alpha|} \bar{w}^{|\alpha|-2} \theta_{j} \frac{\partial}{\partial z_{m}}, \\
& U=\sum_{l, m} \frac{1}{2}\left(U_{m, l}+\overline{U_{l, m}}\right) \frac{\partial}{\partial z_{m}} \wedge \frac{\partial}{\partial \bar{z}_{l}} \\
& U_{m, l}=-\frac{\chi_{1}\left(|s|^{2}\right)}{\chi\left(|s|^{2}\right)}\left\{\sum_{j, k} c_{j k l m} \theta_{j} \bar{\theta}_{k}\right. \\
& +2 \sum_{\alpha, j, k} e_{\alpha j k m} w^{|\alpha|-1} \frac{\alpha_{l}}{|\alpha|} s^{\alpha-\mathbf{1}_{l}} \theta_{j} \bar{\theta}_{k} \\
& \left.+2 \sum_{\alpha, k} d_{\alpha k m}(|\alpha|-1) w^{|\alpha|-2} \frac{\alpha_{l}}{|\alpha|} s^{\alpha-\mathbf{1}_{l}} \eta \bar{\theta}_{k}\right\} \\
& +\sum_{\alpha, \beta, j, k} d_{\alpha k m} \overline{d_{\beta j l}} w^{|\alpha|-1} \bar{w}^{|\beta|-1} s^{\alpha} \bar{s}^{\beta} \theta_{j} \bar{\theta}_{k} .
\end{aligned}
$$

The choice $\chi(t)=\frac{C}{(1-t)^{2}} \exp \left(\frac{1}{t-1}\right)$ for $t<1$ gives $\chi_{1}(t)=-C \exp \left(\frac{1}{t-1}\right)$, so $\chi_{1}(t) / \chi(t)=(1-t)^{2}$ is smooth and bounded, and our vector fields $\Xi, U$ are smooth. We can write

$$
\begin{aligned}
(\theta, \eta s) \wedge \overline{(\theta, \eta s)} & +|w|^{2}(0, \Xi) \wedge \overline{(\theta, \eta s)}+|w|^{2}(\theta, \eta s) \wedge \overline{(0, \Xi)}+|w|^{2}(0, U) \\
& =\left(\theta, \eta s+|w|^{2} \Xi\right) \wedge \overline{\left(\theta, \eta s+|w|^{2} \Xi\right)}+\left(0, U-|w|^{2} \Xi \wedge \bar{\Xi}\right),
\end{aligned}
$$

therefore (3.10) implies the formula in proposition 3.8 with

$$
\begin{aligned}
\tau & =d h_{(0, w s)}\left(\theta, \eta s+|w|^{2} \Xi\right), \\
V & =d h_{(0, w s)}\left(0, U-|w|^{2} \Xi \wedge \Xi\right) .
\end{aligned}
$$

Since $\operatorname{exph}_{z}(\zeta)=h\left(z, \rho(z, \xi), \rho(0, \xi)=\xi+O\left(\xi^{N+1}\right)\right.$ and $\partial \rho_{(0, \xi)}=d \xi+O\left(\xi^{N}\right) d \xi$ by $(2.9)$, we infer that the $(1,0)$-differential of exph at $(x, \zeta) \in T_{X}$ is

$$
\partial \operatorname{exph}_{(x, \zeta)}=d h_{(0, \xi)}+O\left(|\xi|^{N}\right) d \xi
$$

modulo the identification of the tangent spaces $T\left(T_{X}\right)_{(x, \zeta)}$ and $T\left(T_{\mathbb{C}^{n}}\right)_{(0, \xi)}$ given by the coordinates $(z, \xi)$ on $T_{X}$. However, these coordinates are precisely those which realize the splitting $T\left(T_{X}\right)_{(x, \zeta)}=\left(T_{X, x}\right)^{h} \oplus\left(T_{X, x}\right)^{v}$ with respect to the connection $D$. Since $s=\xi+O\left(w^{N} \xi^{N+1}\right)$ and $\xi=\zeta$ at $z=0$, we get

$$
\begin{aligned}
\tau & =\partial \operatorname{exph}_{(x, w \zeta)}\left(\theta^{h}+\eta \zeta^{v}+|w|^{2} \Xi^{v}\right)+O\left(|w|^{N}|\zeta|^{N}\right), \\
V & =\partial \operatorname{exph}_{(x, w \zeta)}\left(U^{v}-|w|^{2} \Xi^{v} \wedge \overline{\Xi^{v}}\right)+O\left(|w|^{N}|\zeta|^{N}\right) .
\end{aligned}
$$

We can drop the terms $O\left(|w|^{N}\right)$ in $\tau$ and $V$ because

$$
\begin{aligned}
\int_{|\zeta|<1} \partial \bar{\partial} \psi\left(\operatorname{exph}_{x}(w \zeta)\right) d \lambda(\zeta) & =\frac{1}{|w|^{2 n}} \int_{|\zeta|<|w|} \partial \bar{\partial} \psi\left(\operatorname{exph}_{x}(\zeta)\right) d \lambda(\zeta) \\
& =O\left(|w|^{-2}\right)
\end{aligned}
$$


by the usual estimates on Lelong numbers (see e.g. [Le69]), thus

$$
\int_{|\zeta|<1} \partial \bar{\partial} \psi\left(\operatorname{exph}_{x}(w \zeta)\right) O\left(|w|^{N}\right) d \lambda(\zeta)=O\left(|w|^{N-2}\right)
$$

After substituting $\zeta$ to $s$ in the formal expression of $\Xi$ and $U$, we get precisely the formulas given in Proposition 3.8. It remains to explain why the remainder term $O\left(|w|^{N-2} \log |w|\right)$ is in fact of the type $O\left(|w|^{N-1}\right)$. To see this, we increase $N$ by two units and estimate the additional terms in the expansions, due to the contribution of all multi-indices $\alpha$ with $|\alpha|=N+1$ or $N+2$. It is easily seen that the additional terms in $\Xi$ and $U$ are $O\left(|w|^{N-1}\right)$, so they are $O\left(|w|^{N+1}\right)$ in $\tau$ and $|w|^{2} V$. The contribution of these terms to $\partial \bar{\partial} \Psi_{(x, w)}$ is thus of the form

$$
\int_{|\zeta|<1} \partial \bar{\partial} \psi\left(\operatorname{exph}_{x}(w \zeta)\right) O\left(|w|^{N+1}\right) d \lambda(\zeta)=O\left(|w|^{N-1}\right)
$$

\section{Approximation theorem and estimates}

A straightforward consequence of our computations is the following approximation theorem for closed positive currents.

TheOREM 4.1. - Let $X$ be a compact $n$-dimensional manifold equipped with a smooth hermitian metric $\omega$. Fix a smooth semipositive $(1,1)$-form $u$ on $X$ such that the Chern curvature tensor $\Theta\left(T_{X}\right) \in \operatorname{Herm}\left(T_{X} \otimes T_{X}\right)$ of $\left(T_{X}, \omega\right)$ satisfies

$$
\left(\Theta\left(T_{X}\right)+u \otimes \operatorname{Id}_{T_{X}}\right)(\theta \otimes \xi, \theta \otimes \xi) \geq 0
$$

for all $\theta, \xi \in T_{X}$ such that $\langle\theta, \xi\rangle=0$. Let $T=\alpha+\frac{i}{\pi} \partial \bar{\partial} \psi$ be a closed real current where $\alpha$ is a smooth closed real $(1,1)$-form and $\psi$ is quasi-psh. Suppose that $T \geq \gamma$ for some real $(1,1)$-form $\gamma$ with continuous coefficients. As $w$ tends to 0 and $x$ runs over $X$, there is a uniform lower bound

$\alpha_{x}[\theta]^{2}+\frac{i}{\pi} \partial \bar{\partial} \Psi_{(x, w)}[\theta, \eta]^{2} \geq \gamma_{x}[\theta]^{2}-\lambda(x,|w|) u_{x}[\theta]^{2}-\delta(|w|)|\theta|^{2}-\frac{1}{\pi} K\left(|\theta||\eta|+|\eta|^{2}\right)$

where $K>0$ is a sufficiently large constant, $\delta(t)$ a continuous increasing function with $\lim _{t \rightarrow 0} \delta(t)=0$, and

$$
\lambda(x, t)=t \frac{\partial}{\partial t}\left(\Psi(x, t)+K t^{2}\right) .
$$

The above derivative $\lambda(x, t)$ is a nonnegative continuous function on $X \times] 0, \varepsilon_{0}$ [ which is increasing in $t$ and such that

$$
\lim _{t \rightarrow 0} \lambda(x, t)=\nu(T, x) \quad(\text { Lelong number of } T \text { at } x) .
$$

In particular, the currents $T_{\varepsilon}=\alpha+\frac{i}{\pi} \partial \bar{\partial} \Psi(\bullet, \varepsilon)$ are smooth closed real currents converging weakly to $T$ as $\varepsilon$ tends to 0 , such that

$$
T_{\varepsilon} \geq \gamma-\lambda(\bullet, \varepsilon) u-\delta(\varepsilon) \omega .
$$


Proof. - It suffices to prove the estimate for $|w|<\varepsilon(\delta)$, with $\delta>0$ fixed in place of $\delta(|w|)$. Also, the estimate is local on $X$. If we change $\psi$ into $\psi+\psi_{0}$ with a smooth function $\psi_{0}$, then $\alpha$ is changed into $\alpha-\frac{i}{\pi} \partial \bar{\partial} \psi_{0}$ and $\Psi$ into $\Psi+\Psi_{0}$, where $\Psi_{0}$ is a smooth function on $X \times \mathbb{C}$ such that $\Psi_{0}(z, w)=\psi_{0}(z)+O\left(|w|^{2}\right)$. It follows that the estimate remains unchanged up to a term $O(1)|\eta|^{2}$. We can thus work on a small coordinate open set $\Omega \subset X$ and choose $\psi_{0}$ such that $\gamma-\left(\alpha-\frac{i}{\pi} \partial \bar{\partial} \psi_{0}\right)$ is positive definite and small at $x$, say equal to $\frac{\delta}{4} \omega_{x}$. After shrinking $\Omega$ and making the change $\psi \mapsto \psi+\psi_{0}$, we may in fact suppose that $T=\alpha+\frac{i}{\pi} \partial \bar{\partial} \psi$ on $\Omega_{x, \delta} \subset \Omega$, where $\alpha$ satisfies $\gamma_{x}-\alpha_{x}=\frac{\delta}{4} \omega_{x}$ and $\gamma-\frac{\delta}{2} \omega \leq \alpha \leq \gamma$ on $\Omega_{x, \delta}$. In particular, $\frac{i}{\pi} \partial \bar{\partial} \psi \geq \gamma-\alpha$ and $\psi$ is plurisubharmonic on $\Omega_{x, \delta}$. All we have to show is that

$$
\frac{i}{\pi} \partial \bar{\partial} \Psi_{(x, w)}[\theta, \eta]^{2} \geq-\lambda(x,|w|) u_{x}[\theta]^{2}-\frac{\delta}{2}|\theta|^{2}-\frac{1}{\pi} K\left(|\theta||\eta|+|\eta|^{2}\right)
$$

for $|w|<w_{0}(\delta)$ small. For this, we apply proposition 3.8 at order $N=2$; order 2 is enough for our purposes since we will neglect all terms in $\partial \bar{\partial} \Psi$ which converge to 0 with $w$, especially all $O(|w|)$ terms. By (3.11), we can neglect all terms of the form $\partial \bar{\partial} \psi\left(\operatorname{exph}_{x}(w \zeta)\right) O\left(|w|^{3}\right)$ under the integral sign. Up to such terms, $\partial \bar{\partial} \psi \cdot\left(\tau \wedge \bar{\tau}+|w|^{2} V\right)_{\operatorname{exph}_{x}(w \zeta)} \chi\left(|\zeta|^{2}\right)$ is equal to

$$
\begin{gathered}
-|w|^{2} \chi_{1}\left(|\zeta|^{2}\right) \operatorname{Re} \sum_{l, m} \frac{\partial^{2} \psi}{\partial \bar{z}_{l} \partial z_{m}}\left\{\frac{\chi\left(|\zeta|^{2}\right)}{-|w|^{2} \chi_{1}\left(|\zeta|^{2}\right)} \bar{\tau}_{l} \tau_{m}+\sum_{j, k} c_{j k l m} \theta_{j} \bar{\theta}_{k}\right. \\
\left.+2 \sum_{|\alpha|=2, k} d_{\alpha k m}(|\alpha|-1) w^{|\alpha|-2} \frac{\alpha_{l}}{|\alpha|} \zeta^{\alpha-\mathbf{1}_{l}} \eta \bar{\theta}_{k}\right\} \\
\geq-|w|^{2} \chi_{1}\left(|\zeta|^{2}\right) \sum_{l, m} \frac{\partial^{2} \psi}{\partial \bar{z}_{l} \partial z_{m}}\left\{\frac{1}{|w|^{2}} \bar{\tau}_{l} \tau_{m}+\sum_{j, k} c_{j k l m}\left(\theta_{j} \bar{\theta}_{k}+\frac{1}{2} \zeta_{j} \eta \bar{\theta}_{k}+\frac{1}{2} \bar{\zeta}_{k} \theta_{j} \bar{\eta}\right)\right\} \\
=-|w|^{2} \chi_{1}\left(|\zeta|^{2}\right) \sum_{l, m} \frac{\partial^{2} \psi}{\partial \bar{z}_{l} \partial z_{m}}\left\{\frac{1}{|w|^{2}} \bar{\tau}_{l} \tau_{m}+\sum_{j, k} c_{j k l m} \tau_{j} \bar{\tau}_{k}\right. \\
\left.-\sum_{j, k} c_{j k l m}\left(\frac{1}{2} \zeta_{j} \eta \bar{\theta}_{k}+\frac{1}{2} \bar{\zeta}_{k} \theta_{j} \bar{\eta}+\zeta_{j} \bar{\zeta}_{k} \eta \bar{\eta}\right)\right\}
\end{gathered}
$$

in view of 2.9 (iii) and of the inequality $0 \leq-\chi_{1} \leq \chi$; in the last equality, we have also used the fact that $\tau=\theta+\eta \zeta+O(|w|)$.

By (3.11), the mixed terms $\theta_{j} \bar{\eta}, \eta \bar{\theta}_{k}$ and the terms $\eta \bar{\eta}$ give rise to contributions bounded below by $-K^{\prime}\left(|\theta||\eta|+|\eta|^{2}\right)$. Hence we get the estimate

$$
\begin{aligned}
& \frac{i}{\pi} \partial \bar{\partial} \Psi_{(x, w)}[\theta, \eta]^{2} \geq \\
& \frac{1}{\pi}|w|^{2} \int_{\mathbb{C}^{n}}-\chi_{1}\left(|\zeta|^{2}\right) \sum_{j, k, l, m} \frac{\partial^{2} \psi}{\partial \bar{z}_{l} \partial z_{m}}\left(\operatorname{exph}_{x}(w \zeta)\right)\left(c_{j k l m}+\frac{1}{|w|^{2}} \delta_{j m} \delta_{k l}\right) \tau_{j} \bar{\tau}_{k} d \lambda(\zeta) \\
& \quad-K^{\prime}\left(|\theta||\eta|+|\eta|^{2}\right) .
\end{aligned}
$$

We need a lemma. 
LEMmA 4.4. - Suppose that the curvature assumption of Theorem 4.1 is satisfied. Then for every $\varepsilon>0$, there is a constant $M_{\varepsilon}>0$ such that

$$
\sum_{j, k, l, m} \frac{1}{2 \pi}\left(c_{j k l m}+M_{\varepsilon} \delta_{j m} \delta_{k l}\right) \tau_{j} \bar{\tau}_{k} \xi_{l} \bar{\xi}_{m}+\sum_{j, k, l} u_{j k} \tau_{j} \bar{\tau}_{k} \xi_{l} \bar{\xi}_{l}+\varepsilon|\tau|^{2}|\xi|^{2} \geq 0
$$

for all tangent vectors $\tau, \xi$.

Let us consider the hermitian form $H$ on $T_{X} \otimes T_{X}$ defined by

$$
H(\tau \otimes \xi, \tau \otimes \xi)=\sum_{j, k, l, m} \frac{1}{2 \pi} c_{j k l m} \tau_{j} \bar{\tau}_{k} \xi_{l} \bar{\xi}_{m}+\sum_{j, k, l} u_{j k} \tau_{j} \bar{\tau}_{k} \xi_{l} \bar{\xi}_{l}+\varepsilon|\tau|^{2}|\xi|^{2} .
$$

Let $\mu$ be the infimum of $H(\tau \otimes \xi, \tau \otimes \xi)$ on the compact set $\{|\tau|=1\} \times\{|\xi|=1\}$. By our curvature assumption we have $H(\tau \otimes \xi, \tau \otimes \xi) \geq \varepsilon$ when $\tau \perp \xi=0$ and $|\tau|=|\xi|=1$, therefore $H(\tau \otimes \xi, \tau \otimes \xi) \geq 0$ on some neighborhood $|\langle\tau, \xi\rangle|<r_{\varepsilon}$ of that set. It follows that

$$
H(\tau \otimes \xi, \tau \otimes \xi)+\frac{|\mu|}{r_{\varepsilon}^{2}}|\langle\tau, \xi\rangle|^{2} \geq 0
$$

for all $|\tau|=|\xi|=1$. Lemma 4.4 follows with $M_{\varepsilon}=|\mu| / r_{\varepsilon}^{2}$.

Let us apply inequality 4.4 to each vector $\xi$ in a basis of eigenvectors of $\partial \bar{\partial} \psi$, multiply by the corresponding (nonnegative) eigenvalue and take the sum. We get

$$
\frac{1}{2 \pi} \sum_{j, k, l, m} \frac{\partial^{2} \psi}{\partial \bar{z}_{l} \partial z_{m}}\left(c_{j k l m}+M_{\varepsilon} \delta_{j m} \delta_{k l}\right) \tau_{j} \bar{\tau}_{k}+\sum_{l} \frac{\partial^{2} \psi}{\partial z_{l} \partial \bar{z}_{l}}\left(u[\tau]^{2}+\varepsilon|\tau|^{2}\right) \geq 0 .
$$

Combining this with (4.3) for $|w|^{2}<1 / M_{\varepsilon}$, we infer

$$
\begin{gathered}
\frac{i}{\pi} \partial \bar{\partial} \Psi_{(x, w)}[\theta, \eta]^{2} \\
\geq-2|w|^{2} \int_{\mathbb{C}^{n}}-\chi_{1}\left(|\zeta|^{2}\right) \sum_{l} \frac{\partial^{2} \psi}{\partial z_{l} \partial \bar{z}_{l}}\left(\operatorname{exph}_{x}(w \zeta)\right)\left(u_{x}[\tau]^{2}+\varepsilon|\tau|^{2}\right) d \lambda(\zeta) \\
-K^{\prime}\left(|\theta||\eta|+|\eta|^{2}\right) \\
\geq-\left\{2|w|^{2} \int_{\mathbb{C}^{n}}-\chi_{1}\left(|\zeta|^{2}\right) \sum_{l} \frac{\partial^{2} \psi}{\partial z_{l} \partial \bar{z}_{l}}\left(\operatorname{exph}_{x}(w \zeta)\right) d \lambda(\zeta)\right\}\left(u_{x}[\theta]^{2}+\varepsilon|\theta|^{2}\right) \\
-K^{\prime \prime}\left(|\theta||\eta|+|\eta|^{2}\right)
\end{gathered}
$$

by (3.11) again, in combination with the equality $\tau=\theta+\eta \zeta+O(|w|))$. The change of variables $\zeta \mapsto s$ defined by $\operatorname{exph}_{x}(w \zeta)=x+w s$ yields $\zeta=s+O\left(w^{2} s^{3}\right)$ by 2.9 (ii), hence choosing $\varepsilon \ll \delta$ small enough we get

$$
\frac{i}{\pi} \partial \bar{\partial} \Psi_{(x, w)}[\theta, \eta]^{2} \geq-\lambda_{\Omega}(x,|w|) u_{x}[\theta]^{2}-\frac{\delta}{3}|\theta|^{2}-K\left(|\theta||\eta|+|\eta|^{2}\right)
$$

where

$$
\lambda_{\Omega}(x,|w|)=2|w|^{2} \int_{\mathbb{C}^{n}}-\chi_{1}\left(|s|^{2}\right) \sum_{l} \frac{\partial^{2} \psi}{\partial z_{l} \partial \bar{z}_{l}}(x+w s) d \lambda(s) .
$$


By definition, the Lelong number $\nu(\psi, x)$ is $\lim _{r \rightarrow 0} \nu(\psi, x, r)$ where

$$
\nu(\psi, x, r)=\frac{1}{\pi^{n-1} r^{2 n-2} /(n-1) !} \int_{B(x, r)} \frac{2}{\pi} \sum_{l} \frac{\partial^{2} \psi}{\partial z_{l} \partial \bar{z}_{l}} d \lambda(z) .
$$

Therefore we have

$$
\nu(\psi, x,|w| r)=\frac{1}{\pi^{n-1} r^{2 n-2} /(n-1) !}|w|^{2} \int_{|s|<r} \frac{2}{\pi} \sum_{l} \frac{\partial^{2} \psi}{\partial z_{l} \partial \bar{z}_{l}}(x+w s) d \lambda(s) .
$$

As $-\chi_{1}\left(|s|^{2}\right)=2 \int_{|s|}^{+\infty} \chi\left(r^{2}\right) r d r$, the Fubini formula gives

$$
\begin{aligned}
\lambda_{\Omega}(x,|w|) & =4|w|^{2} \int_{0}^{+\infty}\left\{\int_{|s|<r} \sum_{l} \frac{\partial^{2} \psi}{\partial z_{l} \partial \bar{z}_{l}}(x+w s) d \lambda(s)\right\} \chi\left(r^{2}\right) r d r \\
& =\frac{2 \pi^{n}}{(n-1) !} \int_{0}^{1} \nu(\psi, x,|w| r) \chi\left(r^{2}\right) r^{2 n-1} d r \\
\lambda_{\Omega}(x, t) & =\int_{\mathbb{C}^{n}} \nu(\psi, x, t|s|) \chi\left(|s|^{2}\right) d \lambda(s) .
\end{aligned}
$$

Hence $\lambda_{\Omega}(x, t)$ is smooth in $(x, t)$, increasing in $t$ and $\lim _{t \rightarrow 0} \lambda_{\Omega}(x, t)=\nu(\psi, x)=$ $\nu(T, x)$, as desired.

The expected estimate (4.2) thus holds on $\Omega$ with $\lambda_{\Omega}(x, t)$ in place of $\lambda(x, t)$; we only have to show that $\lambda_{\Omega}(x, t)-\lambda(x, t)$ is small. By $(4.2), \Psi(x, w)+K|w|^{2}$ is plurisubharmonic in $w$, and so is a convex function of $\log |w|$; since $\Psi(x, t)$ is bounded above as $t$ tends to 0 , the sum $\Psi(x, t)+K t^{2}$ which is convex in log $t$ must be increasing in $t$. Therefore

$$
\lambda(x, t)=\frac{\partial}{\partial \log t}\left(\Psi(x, t)+K t^{2}\right)
$$

is a nonnegative increasing function of $t$. When we put $\theta=0$, Proposition 3.8 gives $\Xi=V=0$ and $\tau=\partial \operatorname{exph}_{(x, w \zeta)}\left(\eta \zeta^{v}\right)$, thus

$$
\begin{aligned}
\frac{\partial^{2} \Psi}{\partial w \partial \bar{w}}(x, w) & =\int_{\zeta \in T_{X, x}} \partial \bar{\partial} \psi_{\operatorname{exph}_{x}(w \zeta)}[\zeta]^{2} \chi\left(|\zeta|^{2}\right) d \lambda(\zeta)+O\left(|w|^{N-1}\right) \\
& =\int_{\mathbb{C}^{n}} \partial \bar{\partial} \psi_{x+w s}[s]^{2} \chi\left(|s|^{2}\right) d \lambda(s)+O(1),
\end{aligned}
$$

again by the change of variable $\operatorname{exph}_{x}(w \zeta)=x+w s$, for which $\zeta=s+O\left(w^{2} s^{3}\right)$. Since $\partial^{2} / \partial w \partial \bar{w}=\left(t^{-1} \partial / \partial t\right) \circ(t \partial / \partial t)$ for a function of $w$ depending only on $t=|w|$, a multiplication by $t$ followed by an integration implies

$$
\begin{aligned}
t \frac{\partial \Psi(x, t)}{\partial t} & =t \frac{\partial}{\partial t} \int_{\mathbb{C}^{n}} \psi(x+t s) \chi\left(|s|^{2}\right) d \lambda(s)+O\left(t^{2}\right) \\
& =\int_{\mathbb{C}^{n}} \nu(\psi, x, t|s|) \chi\left(|s|^{2}\right) d \lambda(s)+O\left(t^{2}\right)=\lambda_{\Omega}(x, t)+O\left(t^{2}\right)
\end{aligned}
$$

here, we used the well known fact that $\nu(\psi, x, t)$ is equal to the derivative $\partial / \partial \log t$ of the mean value of $\psi$ on the sphere $S(x, t)$. Hence $\lambda_{\Omega}(x, t)-\lambda(x, t)=O\left(t^{2}\right)$ and 
the first estimate in 4.1 follows. Now, it is clear that $\psi_{\varepsilon}$ converges to $\psi$ in $L_{\text {loc }}^{1}$, so $T_{\varepsilon}$ converges weakly to $T$; note also that $\psi_{\varepsilon}+K \varepsilon^{2}$ is increasing in $\varepsilon$ by the above arguments. The proof is complete.

REMARK 4.6. - An integration of the first equality in (4.5) also shows that

$$
\Psi(x, t)=\int_{|s|<1} \psi(x+t s) \chi\left(|s|^{2}\right) d \lambda(s)+O\left(t^{2}\right)
$$

relatively to the system of normal coordinates at $x$ defined in $\S 2$.

REMARK 4.7. - The estimates obtained in Theorem 4.1 can be slightly improved by setting

$$
\widetilde{\Psi}(x, w)=\Psi(x, w)+|w|, \quad \widetilde{\lambda}(x, t)=t \frac{\partial}{\partial t}(\widetilde{\Psi}(x, t)) .
$$

Indeed $\widetilde{\lambda}(x, t)=\lambda(x, t)+t-2 K t^{2}$ is increasing in $t$ and larger than $\lambda(x, t)$ for $t$ small, hence $\widetilde{\Psi}(x, w)$ is convex and increasing in $\log |w|$, while

$$
\partial \bar{\partial} \widetilde{\Psi}_{(x, w)}[\theta, \eta]^{2}=\partial \bar{\partial} \Psi_{(x, w)}[\theta, \eta]^{2}+\frac{|\eta|^{2}}{4|w|} .
$$

Since $K|\theta||\eta| \leq 2 K^{2}|w||\theta|^{2}+|\eta|^{2} /(8|w|)$, we get for $|w|<\varepsilon_{0}$ small enough a lower bound of the form

$$
\alpha_{x}[\theta]^{2}+\frac{i}{\pi} \partial \bar{\partial} \widetilde{\Psi}_{(x, w)}[\theta, \eta]^{2} \geq \gamma_{x}[\theta]^{2}-\widetilde{\lambda}(x,|w|) u_{x}[\theta]^{2}-\widetilde{\delta}(|w|)|\theta|^{2},
$$

where $\lim _{t \rightarrow 0} \widetilde{\lambda}(x, t)=\nu(T, x)$ and $\lim _{t \rightarrow 0} \widetilde{\delta}(t)=0, \widetilde{\delta}$ being continuous and increasing.

\section{Compact manifolds with partially semipositive curvature}

In case the curvature of the ambient manifold $X$ satisfies suitable semipositivity assumptions, our smoothing theorem 4.1 yields interesting geometric consequences.

DeFinition 5.1. - We say that $X$ has partially semipositive curvature (in the sense of Griffiths) if there exists a hermitian metric $\omega$ on $X$ such that the associated Chern curvature tensor $\Theta\left(T_{X}\right)$ satisfies

$$
\Theta\left(T_{X}\right)(\theta \otimes \xi, \theta \otimes \xi) \geq 0
$$

for all $\theta, \xi \in T_{X}$ with $\langle\theta, \xi\rangle=0$.

The standard Griffiths semipositivity assumption for $T_{X}$ would require that the above inequality is satisfied for all pairs $\theta, \xi \in T_{X}$. This is the case if $X$ is a compact complex homogeneous manifold, since the tangent bundle is then generated by global sections; a fortiori, such manifolds satisfy the property in Def. 5.1. However, the partial semipositivity condition is substantially weaker. In 
fact, every complex curve has partially semipositive curvature, but a curve has a metric of semipositive curvature if and only if it is of genus 0 or 1 . This observation gives rise immediately to higher dimensional examples; in fact let $X=\mathbb{P}^{1} \times C$ where $C$ is a curve of genus $g \geq 2$, equipped with its unique metric of constant curvature -1 . The Fubini-Study metric on $\mathbb{P}^{1}$ has curvature +2 , hence we have

$$
\left\langle\Theta\left(T_{X}\right)(\theta \otimes \xi), \theta \otimes \xi\right\rangle=2\left|\theta_{1}\right|^{2}\left|\xi_{1}\right|^{2}-\left|\theta_{2}\right|^{2}\left|\xi_{2}\right|^{2},
$$

where the subscripts 1,2 denote respectively the first and second projection of a tangent vector; clearly the difference is nonnegative when $\left\langle\theta_{1}, \xi_{1}\right\rangle+\left\langle\theta_{2}, \xi_{2}\right\rangle=0$, thus $\mathbb{P}^{1} \times C$ has partially semipositive curvature. It would be interesting to have a more algebraic understanding of the notion of partial semipositivity. This is certainly a very difficult problem; recall in this context the deep unsolved conjecture of Griffiths that ampleness is equivalent to Griffiths positivity.

The following results were already obtained in [De92] under the assumption that $T_{X}$ is nef (i.e. $\mathcal{O}_{T_{X}}(1)$ is nef over $P\left(T_{X}^{\star}\right)$ ). It is well known that Griffiths semipositivity implies nefness, and that the converse is not true; however, if Griffiths' conjecture holds, nefness would be equivalent to the fact that the curvature tensor $\Theta\left(T_{X}\right)$ can be made larger than $-\varepsilon$ for every $\varepsilon>0$ (see [DPS92]). The above examples show that partial semipositivity covers different situations.

Proposition 5.2. - Let $(X, \omega)$ be a compact hermitian manifold with partially semipositive curvature.

(i) For any closed real $(1,1)$-current $T$ on $X$ such that $T \geq \gamma$ for some continuous real $(1,1)$-form $\gamma$ on $X$, there is a family $T_{\varepsilon}$ of smooth approximations in the same $\partial \bar{\partial}$-cohomology class as $T$ and converging weakly to $T$ as $\varepsilon$ tends to 0 , such that $T_{\varepsilon} \geq \gamma-\delta_{\varepsilon} \omega$ with $\lim _{\varepsilon \rightarrow 0} \delta_{\varepsilon}=0$.

(ii) Every closed positive $(1,1)$-current $T$ is numerically effective in the sense of [De92] ; in particular, if $X$ is Kähler, the De Rham cohomology class $\{T\}$ satisfies $\int_{Y}\{T\}^{p} \geq 0$ for every $p$-dimensional analytic subset $Y \subset X$.

Proof. - (i) follows immediately from Theorem 4.1 by taking $u=0$.

To get (ii), we apply (i) with $\gamma=0$. We then obtain $T_{\varepsilon} \geq-\delta_{\varepsilon} \omega$. Let $\omega_{0}$ be a Kähler metric on $X$ ( $\omega_{0}$ need not be related to $\omega$ ). Multiplying $\omega_{0}$ by a suitable constant we get $\omega_{0} \geq \omega$ and so $T_{\varepsilon}+\delta_{\varepsilon} \omega_{0} \geq 0$. As $\{T\}=\left\{T_{\varepsilon}\right\}$, this implies

$$
\int_{Y}\left(\{T\}+\delta_{\varepsilon}\left\{\omega_{0}\right\}\right)^{p}=\int_{Y}\left(T_{\varepsilon}+\delta_{\varepsilon} \omega_{0}\right)^{p} \geq 0,
$$

thus $\int_{Y}\{T\}^{p} \geq 0$ in the limit.

Proposition 5.2 implies that a compact complex manifold with partially semipositive curvature must be minimal in the sense that it does not contain any divisor $D$ which can be blown down to some lower dimensional variety. Indeed, in the latter case, there is always a curve $C \subset D$ such that $D \cdot C=\int_{C}\{D\}<0$. 
Proposition 5.3. - Let $X$ be a compact complex manifold with partially semipositive curvature. Then $X$ is Kähler if and only if $X$ is in the Fujiki class $\mathcal{C}$ (= class of complex varieties which are bimeromorphic to Kähler manifolds), and $X$ is projective if and only if $X$ is Moishezon.

Proof. - Let $X$ be a manifold in the Fujiki class $\mathcal{C}$ such that $T_{X}$ has partially semipositive curvature. We know by Fujiki [Fu78] that $X$ has a smooth Kähler modification $\mu: \widetilde{X} \rightarrow X$. Let $\beta$ be a Kähler metric on $\widetilde{X}$ and let $T=\mu_{\star} \beta$ be the direct image current. If $\omega$ is a hermitian metric on $X$, we have $\beta \geq c \mu^{\star} \omega$ on $\widetilde{X}$ for some small constant $c>0$, thus by taking the direct image we get $T=\mu_{\star} \beta \geq c \omega$ on $X$. Now Proposition 5.2 (i) produces a smooth approximation $T_{\varepsilon}$ of $T$ such that $T_{\varepsilon} \geq c \omega-\delta_{\varepsilon} \omega \geq \frac{c}{2} \omega$ for $\varepsilon$ small enough. Thus $T_{\varepsilon}$ is a Kähler metric and $X$ is Kähler. In particular, if $X$ is Moishezon, we can take $\beta$ to be the curvature form of an ample line bundle $\mathcal{O}(D)$ over $\widetilde{X}$. Then $T$ and $T_{\varepsilon}$ are cohomologous to $\mu_{\star}([D])=\left[D^{\prime}\right]$ where $D^{\prime}=\mu(D)$ is the image of $D$ in $X$. This implies that $\mathcal{O}\left(D^{\prime}\right)$ is ample and therefore $X$ is projective algebraic (we could also have applied the well-known result of Moishezon that every Kähler Moishezon manifold is projective algebraic). The converse implications are trivial.

\section{Singularity attenuation process for closed $(1,1)$-currents}

If $T$ is a closed positive or almost positive current on a complex manifold $X$, we denote by $E_{c}(T)$ the $c$-upperlevel set of Lelong numbers:

$$
E_{c}(T)=\{x \in X ; \nu(T, x) \geq c\}, \quad c>0 .
$$

A well-known theorem of [Siu74] asserts that $E_{c}(T)$ is a closed analytic subset of $X$. A combination of Theorem 4.1 with Kiselman's singularity attenuation technique yields the following partial regularization process. The sets $E_{c}(T)$ appear precisely to be the obstructions to smoothing when no loss of positivity is admitted.

Theorem 6.1. - Let $T$ be a closed almost positive $(1,1)$-current and let $\alpha$ be a smooth real $(1,1)$-form in the same $\partial \bar{\partial}$-cohomology class as $T$, i.e. $T=\alpha+\frac{i}{\pi} \partial \bar{\partial} \psi$ where $\psi$ is an almost psh function. Let $\gamma$ be a continuous real $(1,1)$-form such that $T \geq \gamma$. Suppose that $T_{X}$ is equipped with a smooth hermitian metric $\omega$ such that the Chern curvature form satisfies

$$
\left(\Theta\left(T_{X}\right)+u \otimes \operatorname{Id}_{T_{X}}\right)(\theta \otimes \xi, \theta \otimes \xi) \geq 0 \quad \forall \theta, \xi \in T_{X} \quad \text { with }\langle\theta, \xi\rangle=0,
$$

for some continuous nonnegative $(1,1)$-form $u$ on $X$. Then for every $c>0$, there is a family of closed almost positive $(1,1)$-currents $\left.T_{c, \varepsilon}=\alpha+\frac{i}{\pi} \partial \bar{\partial} \psi_{c, \varepsilon}, \varepsilon \in\right] 0, \varepsilon_{0}[$, such that $\psi_{c, \varepsilon}$ is smooth on $X \backslash E_{c}(T)$, increasing with respect to $\varepsilon$, and converges to $\psi$ as $\varepsilon$ tends to 0 (in particular, the current $T_{c, \varepsilon}$ is smooth on $X \backslash E_{c}(T)$ and converges weakly to $T$ on $X)$, and such that

(i) $T_{c, \varepsilon} \geq \gamma-\min \left\{\lambda_{\varepsilon}, c\right\} u-\delta_{\varepsilon} \omega$ where:

(ii) $\lambda_{\varepsilon}(x)$ is an increasing family of continuous functions on $X$ such that $\lim _{\varepsilon \rightarrow 0} \lambda_{\varepsilon}(x)=\nu(T, x)$ at every point, 
(iii) $\delta_{\varepsilon}$ is an increasing family of positive constants such that $\lim _{\varepsilon \rightarrow 0} \delta_{\varepsilon}=0$,

(iv) $\nu\left(T_{c, \varepsilon}, x\right)=(\nu(T, x)-c)_{+}$at every point $x \in X$.

Proof. - Following an idea of Kiselman [Ki79], we let $\psi_{c, \varepsilon}$ be the Legendre transform

$$
\psi_{c, \varepsilon}(x)=\inf _{|w|<1}\left(\widetilde{\Psi}(x, \varepsilon w)+\frac{\varepsilon}{1-|w|^{2}}-c \log |w|\right),
$$

where $\widetilde{\Psi}(x, w)=\Psi(x, w)+|w|$ is the function defined in Remark 4.7. It is clear that $\psi_{c, \varepsilon}$ is increasing in $\varepsilon$ and that

$$
\lim _{\varepsilon \rightarrow 0} \psi_{c, \varepsilon}(x)=\widetilde{\Psi}\left(x, 0_{+}\right)=\Psi\left(x, 0_{+}\right)=\psi(x) .
$$

Moreover, as $\widetilde{\Psi}(x, w)$ is convex and increasing in $t=\log |w|$, the function

$$
t \longmapsto \Phi_{c, \varepsilon}(x, t):=\widetilde{\Psi}(x, \varepsilon t)+\frac{\varepsilon}{1-t^{2}}-c \log t
$$

is strictly convex in $\log t$ and tends to $+\infty$ as $t$ tends to 1 . It follows that the infimum is attained for $t=t_{0}(x) \in[0,1$ [ given either by the zero of the $\partial / \partial \log t$ derivative:

$$
\widetilde{\lambda}(x, \varepsilon t)+\frac{2 \varepsilon t^{2}}{\left(1-t^{2}\right)^{2}}-c=0
$$

when $\nu(T, x)=\lim _{t \rightarrow 0} \tilde{\lambda}(x, t)<c$, or by $t_{0}(x)=0$ when $\nu(T, x) \geq c$ (in which case the above strictly convex function is increasing on the whole interval $[0,1[)$. Since the $\partial / \partial \log t$ derivative is itself strictly increasing in $t$, the implicit function theorem shows that $t_{0}(x)$ depends smoothly on $x$ on $X \backslash E_{c}(T)=\{\nu(T, x)<c\}$, hence $\psi_{c, \varepsilon}(x)=\Phi_{c, \varepsilon}\left(x, t_{0}(x)\right)$ is actually smooth on $X \backslash E_{c}(T)$.

Now, fix a point $x \in X \backslash E_{c}(T)$ and $t_{1}>t_{0}(x)$. For all $z$ in a neighborhood $V$ of $x$ we still have $t_{0}(z)<t_{1}$, thus

$$
\psi_{c, \varepsilon}(z)=\inf _{|w|<t_{1}}\left(\widetilde{\Psi}(z, \varepsilon w)+\frac{\varepsilon}{1-|w|^{2}}-c \log |w|\right) \quad \text { on } V .
$$

By (4.8), all functions involved in that infimum have a complex Hessian in $(z, w)$ bounded below by

$$
\gamma_{z}-\alpha_{z}-\tilde{\lambda}\left(z, \varepsilon t_{1}\right) u_{z}-\widetilde{\delta}\left(\varepsilon t_{1}\right) \omega_{z}
$$

Hence $\partial \bar{\partial} \psi_{c, \varepsilon}$ has the same lower bound (this is an easy consequence of Kiselman's principle [Ki78] that an infimum $\inf _{w} u(z, w)$ of plurisubharmonic functions depending only on Re $w$ is plurisubharmonic in $z)$. By taking $t_{1}$ arbitrarily close to $t_{0}(x)$ and by shrinking $V$, the lower bound comes arbitrary close to $\gamma_{x}-\alpha_{x}-\tilde{\lambda}\left(x, \varepsilon t_{0}(x)\right) u_{x}-\widetilde{\delta}\left(\varepsilon t_{0}(x)\right) \omega_{x} \geq \gamma_{x}-\alpha_{x}-\min \{\tilde{\lambda}(x, \varepsilon), c\} u_{x}-\widetilde{\delta}(\varepsilon) \omega_{x}$, because $\widetilde{\lambda}\left(x, \varepsilon t_{0}(x)\right)=c-2 \varepsilon t_{0}(x)^{2} /\left(1-t_{0}(x)^{2}\right)^{2} \leq c$ and $\widetilde{\lambda}(x, t), \widetilde{\delta}(t)$ are increasing in $t$. Therefore we get

$$
\alpha+\frac{i}{\pi} \partial \bar{\partial} \psi_{c, \varepsilon} \geq \gamma-\min \{\tilde{\lambda}(\bullet, \varepsilon), c\} u-\widetilde{\delta}(\varepsilon) \omega
$$


on $X \backslash E_{c}(T)$. However, as the lower bound is a continuous $(1,1)$-form and as $\psi_{c, \varepsilon}$ is quasi-psh, it is immediate to check that the lower bound extends to $X$ by continuity. Hence properties 6.1 (i), (ii), (iii) are proved.

Remark 4.6 finally shows that $\psi_{c, \varepsilon}$ differs locally from Kiselman's usual Legendre transform only by bounded terms, thus

$$
\nu\left(\psi_{c, \varepsilon}, x\right)=(\nu(\psi, x)-c)_{+}
$$

at every point $x \in X$ by Kiselman's results [Ki78], [Ki79]. Therefore property 6.1 (iv) also holds.

\section{References}

[De82] Demailly, J.-P. — Estimations $L^{2}$ pour l'opérateur $\bar{\partial}$ d'un fibré vectoriel holomorphe semi-positif au dessus d'une variété kählérienne complète, Ann. Sci. Ec. Norm. Sup., 15 (1982), 457-511.

[De90] Demailly, J.-P. - Singular hermitian metrics on positive line bundles, Proceedings of the Bayreuth conference "Complex algebraic varieties", April 2-6, 1990, edited by K. Hulek, T. Peternell, M. Schneider, F. Schreyer, Lecture Notes in Math. $\mathrm{n}^{\circ}$ 1507, Springer-Verlag, 1992.

[De91a] Demailly, J.-P. — A numerical criterion for very ample line bundles, Prépublication $\mathrm{n}^{\circ} 153$, Institut Fourier, Univ. Grenoble I, September 1991, to appear in J. Differential Geom.

[De91b] Demailly, J.P. - Monge-Ampère operators, Lelong numbers and intersection theory, Prépub. Inst. Fourier $n^{\circ} 173$, Mai 1991, 70p, to appear in the Proceedings "Complex Analysis and Geometry", CIRM, Trento (Italy), edited by V. Ancona and A. Silva.

[De92] Demailly, J.-P. - Regularization of closed positive currents and Intersection Theory, J. Alg. Geom., 1 (1992), 361-409.

[DPS91] Demailly, J.P., Peternell, Th., Schneider, M. - Compact complex manifolds with numerically effective tangent bundles, Prépub. Inst. Fourier $\mathrm{n}^{\circ} 184$, Octobre 1991, 48p.

[Fu78] A. FUJIKI. - Closedness of the Douady spaces of compact Kähler spaces, Publ. R.I.M.S., Kyoto Univ., 14 (1978), 1-52.

[Hö66] HöRmANDER, L. - An introduction to Complex Analysis in several variables, North-Holland Math. libr., vol.7, Amsterdam, London, 1966, third edition,1991.

[Ki78] KisElman, C.O. - The partial Legendre transformation for plurisubharmonic functions, Invent. Math., 49 (1978), 137-148.

[Ki79] Kiselman, C.O. - Densité des fonctions plurisousharmoniques, Bull. Soc. Math. France, 107 (1979), 295-304.

[Le57] LELONG, P. - Intégration sur un ensemble analytique complexe, Bull. Soc. Math. France, 85 (1957), 239-262.

[Le69] Lelong, P. _ Plurisubharmonic functions and positive differential forms, Gordon and Breach, New-York, and Dunod, Paris, 1969.

[Siu74] SIU, Y.T. - Analyticity of sets associated to Lelong numbers and the extension of closed positive currents, Invent. Math., 27 (1974), 53-156.

(January 22, 1993) 